\title{
Transcranial Direct Current Stimulation in the Treatment of Subacute Post-Stroke Thalamic Aphasia
}

\author{
William Campanella ${ }^{1,2}$, Riccardo Pedrini $^{1,2}$, Lucilla Vestito ${ }^{1}$, Lucio Marinelli1,2, Carlo Trompetto ${ }^{1,2}$, Laura Mori ${ }^{1,2}$ \\ ${ }^{1}$ IRCCS Ospedale Policlinico San Martino, Genoa, Italy
}

${ }^{2}$ Department of Neurosciences, Rehabilitation, Ophthalmology, Genetics, Maternal and Child Health (DiNOGMI), University of Genoa, Genoa, Italy

\section{Doi: 10.12890/2020_001794 - European Journal of Case Reports in Internal Medicine - ๑ EFIM 2020}

Received: $11 / 06 / 2020$

Accepted: $18 / 06 / 2020$

Published: 03/09/2020

How to cite this article: Campanella W, Pedrini R, Vestito L, Marinelli L, Trompetto C, Mori L. Transcranial direct current stimulation in the treatment of subacute post-stroke thalamic aphasia. EJCRIM 2020;7: doi:10.12890/2020_001794.

Conflicts of Interests: The Authors declare that there are no competing interests.

This article is licensed under a Commons Attribution Non-Commercial 4.0 License

\section{ABSTRACT}

Background: Although many studies have demonstrated the effectiveness of transcranial direct current stimulation (tDCS) in improving speech recovery in post-stroke aphasia, as far as we know patients affected by thalamic aphasia have never been investigated.

Patient and method: A 65-year-old man with severe non-fluent aphasia due to a left thalamic haemorrhagic stroke underwent intensive daily speech therapy combined with tDCS.

Results: The patient showed progressive improvement with almost complete recovery of his speech disorder, behavioural disinhibition and apathy.

Conclusions: Our findings suggest that tDCS with concurrent speech therapy can be useful in patients with subcortical stroke lesions.

\section{LEARNING POINTS}

- The thalamus has a central role in the development of the higher functions, such as memory, regulation of behaviour and emotions, and language elaboration.

- Transcranial direct current stimulation (tDCS) applied to the cerebral cortex is a promising tool for improving language recovery in patients with post-stroke aphasia.

- Combined treatment with tDCS and speech and language therapy (SLT) was effective in a patient with subacute thalamic aphasia, who showed improvement in all aspects of linguistic and behavioural communication.

\section{KEYWORDS}

Transcranial direct current stimulation, aphasia, cerebral haemorrhage, speech therapy, rehabilitation

\section{CASE DESCRIPTION}

We present the case of a 65-year-old, right-handed man, with 8 years of education, who experienced haemorrhagic stroke in the left thalamus associated with endoventricular blood outflow and perilesional oedema, as shown by magnetic resonance imaging (Fig. 1).

The patient was admitted to the neurology department where clinical examination demonstrated right hemiplegia associated with severe expressive aphasia. He underwent 20 days of daily physiotherapy and speech therapy. He was then transferred to our neurorehabilitation unit for intensive rehabilitation.

At admission to the neurorehabilitation unit (TO), the patient's language deficit was explored using the Neuropsychological Examination for Aphasia (ENPA). ENPA results are given in Table 1. The Boston Naming Test-Short Form (BNT-SF) showed very poor patient performance but 


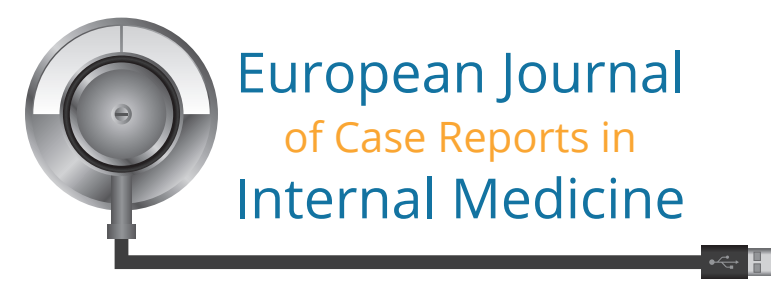

well-preserved general cognitive ability. The patient performed normally on Raven's Coloured Progressive Matrices visual search behaviour and on the Corsi Block-Tapping Test (Table 2).

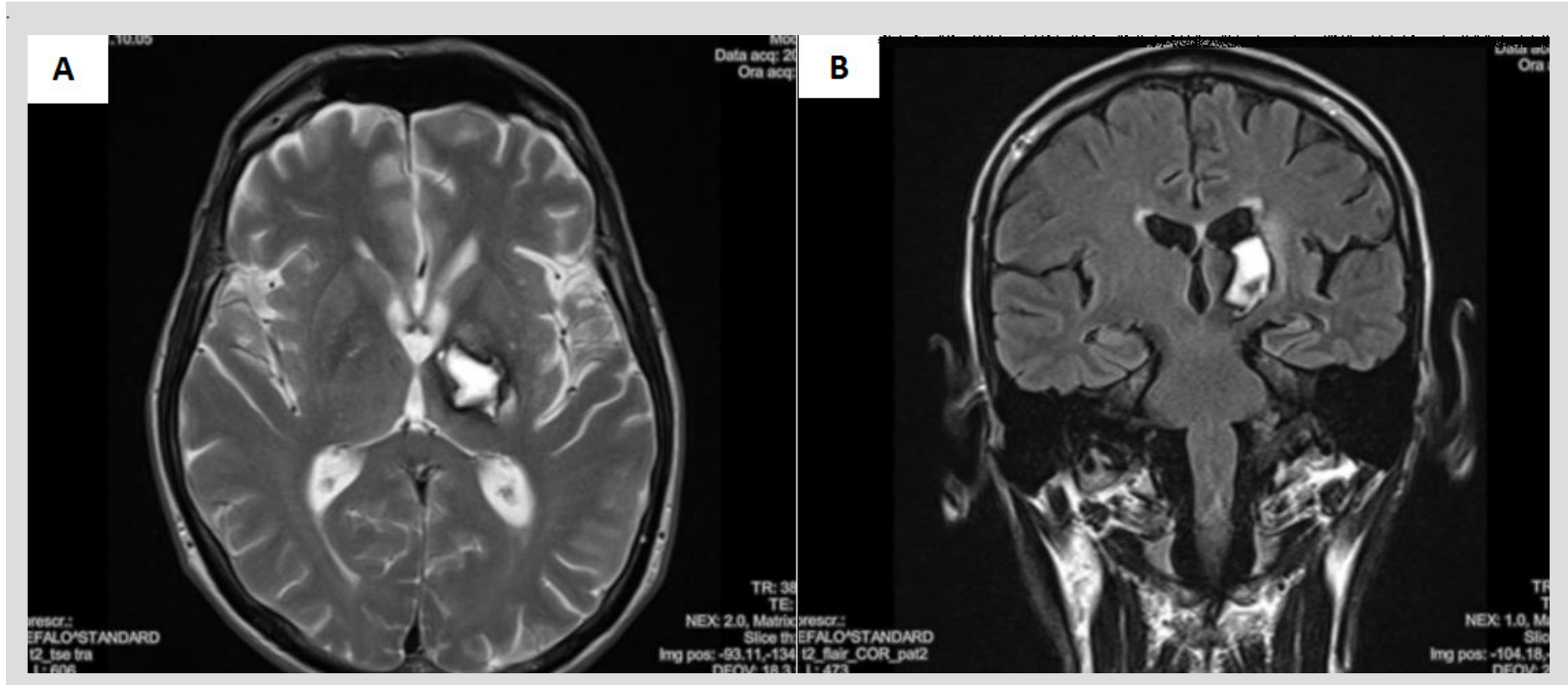

Figure 1. Cerebral magnetic resonance imaging performed in the acute phase. T2-weighted axial (A) and T2-flair sagittal (B) slices disclosed a hyperintense area in the left thalamus

\begin{tabular}{|c|c|c|c|c|c|c|c|}
\hline Task & TO & Corrected score & T1 & Corrected score & T2 & Corrected score & Cut-off \\
\hline \multicolumn{8}{|l|}{ Repetition } \\
\hline Words & 2 & 1.8 & 2 & 1.8 & 5 & 4.8 & 8.8 \\
\hline Non-words & 0 & 0.2 & 0 & 0.2 & 2 & 2.2 & 2.0 \\
\hline Sentences & 0 & 0 & 0 & 0 & 1 & 1 & 3.0 \\
\hline \multicolumn{8}{|l|}{ Reading } \\
\hline Words & 8 & 5.4 & 8 & 5.4 & 10 & 7.4 & 6.4 \\
\hline Non-words & 1 & 1 & 1 & 1 & 4 & 4 & 4.0 \\
\hline Sentences & 1 & 0.9 & 1 & 0.9 & 2 & 1.9 & 1.3 \\
\hline \multicolumn{8}{|l|}{ Writing } \\
\hline Words & 2 & 1.9 & 2 & 1.9 & 5 & 4.9 & 6.3 \\
\hline Non-words & 0 & 0 & 0 & 0 & 1 & 0.6 & 1.4 \\
\hline Sentences & 0 & 0 & 0 & 0 & 0 & 0 & 0.6 \\
\hline \multicolumn{8}{|l|}{ Denomination } \\
\hline Oral nouns & 3 & 3 & 3 & 3 & 9 & 9 & 8.2 \\
\hline Written nouns & 1 & 1.3 & 1 & 1.3 & 4 & 4.3 & 2.7 \\
\hline Oral verbs & 4 & 3.9 & 4 & 3.9 & 9 & 8.9 & 6.1 \\
\hline Written verbs & 2 & 1.8 & 2 & 1.8 & 3 & 2.8 & 3.0 \\
\hline Colours & 4 & 4 & 4 & 4 & 5 & 5 & 4.0 \\
\hline \multicolumn{8}{|l|}{ Comprehension } \\
\hline Auditory words & 18 & 17.4 & 18 & 17.4 & 20 & 19.4 & 18.4 \\
\hline Visual words & 15 & 15.4 & 15 & 15.4 & 17 & 17.4 & 17.0 \\
\hline Auditory sentences & 9 & 9.3 & 9 & 9.3 & 14 & 14.3 & 11.6 \\
\hline Visual sentences & 3 & 2.9 & 3 & 2.9 & 13 & 12.9 & 11.3 \\
\hline
\end{tabular}

Table 1. Results of the ENPA test at different follow-up times 
The patient was classified as having non-fluent aphasia: his spontaneous speech was very poor with short sentences and frequent wordfinding difficulties, oral denomination of nouns and verbs was deficient, and anomia and semantic paraphasia were present. Auditory and visual comprehension of words was only slightly impaired, but auditory comprehension of sentences appeared compromised. Reading aloud was preserved despite some phonetic distortions. Reading and writing non-words and sentences was impaired. There was no bucco-linguofacial, ideomotor, ideational or constructive apraxia. The patient also showed personality changes, behavioural disinhibition and apathy.

\begin{tabular}{|l|l|l|}
\hline Test & Score & Cut-off \\
\hline $\begin{array}{l}\text { Raven's Coloured } \\
\text { Progressive Matrices }\end{array}$ & $30 / 36$ & $\geq 18$ \\
\hline $\begin{array}{l}\text { Spinnler and Tognoni's } \\
\text { Visual Search Test }\end{array}$ & $45 / 60$ & $\geq 30$ \\
\hline Corsi Block-Tapping Test & $5 / 10$ & $\geq 3.75$ \\
\hline $\begin{array}{l}\text { Boston Naming Test- } \\
\text { Short Form }\end{array}$ & $3 / 15$ & \\
\hline
\end{tabular}

Table 2. Neuropsychological assessment at TO

Methods and Procedures

The patient initially underwent conventional rehabilitation. However, after 2 weeks (T1), despite a slight improvement in test performance, his scores had not reached the normal cut-off values (Table 1).

In light of his high level of motivation and the substantial integrity of his verbal receptive ability, we decided to treat the patient with transcranial direct current stimulation (tDCS) while he was also receiving speech and language therapy (SLT). tDCS is a safe and relatively painless technique, where a low-intensity direct current (1-2 $\mathrm{mA}$ ) is applied over the scalp to modulate cortical excitability. The patient and his caregiver were both informed of treatment details, the possible beneficial effects and the possible risks, and informed consent was obtained.

Stimulation electrodes measuring $5 \mathrm{~cm} \times 5 \mathrm{~cm}$ were placed on the patient's scalp and held in place with a cloth cap. The most common configuration, as supported by functional magnetic resonance imaging results, involves the application of anodal-tDCS (A-tDCS) over the left prefrontal inferior gyrus with the reference electrode positioned over the contralateral supraorbital region ${ }^{[1]}$. In our patient, to target Broca's area, the active electrode was placed between T3-Fz and F7-Cz (according to the EEG 10-20 system), while the reference electrode was over the contralateral supraorbital region. A-tDCS was applied with a constant stimulation intensity of $1.5 \mathrm{~mA}$.

In addition to A-tDCS, the patient underwent an intensive daily session of SLT lasting 20 minutes, for 10 consecutive days (5 days a week). SLT consisted of denomination tasks consisting of 40 two-dimensional black and white pictures, divided into 20 higher frequency use stimuli (e.g., plate, flower, etc.), 10 lower frequency use stimuli (skyscraper, seahorse, etc.) and 10 actions (applauding, pouring, etc.). Each stimulus was presented for 25 seconds, with a lag time of 5 seconds between one stimulus and the next. The patient was required to correctly name all the stimuli and, in case of anomia, no help was given by the examiner. To avoid the learning effect, during the second week, the stimuli were modified maintaining the same subdivisions; moreover, stimuli presented during the training session were different to those included in the ENPA test or in the BNT-SF. The procedure was well tolerated and the patient was able to complete the protocol. He showed progressive improvement in his performance and, at the end of treatment (T2), was again evaluated with the ENPA test (Table 1) and BNT-SF (13/15), demonstrating improvement in all fields explored, and succeeding in naming $85 \%$ of the stimuli (Fig. 2).

Formal evaluation of language at T2 showed a patient with good intentional communication. He was able to express his personal needs and manage informative conversations with other subjects. Spontaneous language was characterized by good syntactic structure and rare anomia was overcome with correct circumlocutions. His speech was informative and appropriate to his level of education. The positive results were extended to other aspects of the aphasic syndrome. The oral and written denomination tests were within the normal range, although still with a few errors. Oral and written comprehension of words and sentences was normal, as was reading words, non-words and sentences. The behavioural disinhibition and apathy which affected the patient at T0 and T1 were no longer present.

\section{DISCUSSION}

The primary function of the thalamus is to process most of the information reaching the cerebral cortex from the spinal cord and the other parts of the central nervous system. The thalamus also has a central role in the development of the higher functions, such as memory, regulation of behaviour and emotions, and language elaboration. The advent of modern techniques in neuroimaging has led to better 


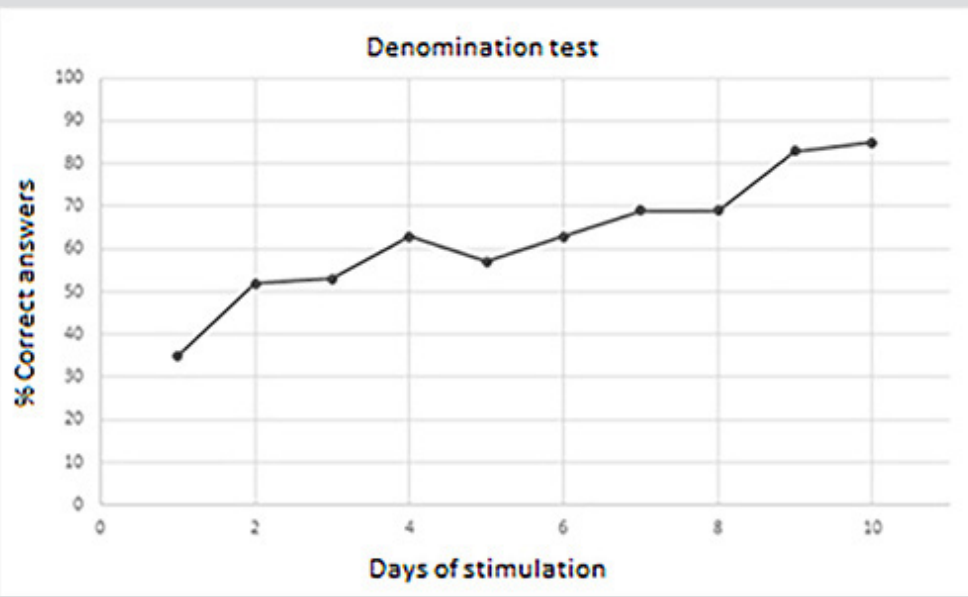

Figure 2. Progressive improvement in denomination test performance during transcranial direct current stimulation (tDCS) and speech and language therapy (SLT)

understanding of the role the thalamus and other subcortical structures in language disturbance ${ }^{[2,3]}$. The thalamus projects to all areas of the neocortex including those in the frontal and temporal regions that are associated with language, with direct and reciprocal connections between subregions of Broca's area and subcortical structures ${ }^{[2,3]}$.

A recent review ${ }^{[4]}$ shows that tDCS is effective for treating post-stroke aphasia when applied in combination with SLT in the chronic post-stroke phase, although the benefits of its application in the subacute phase are more difficult to demonstrate since the real effect of stimulation can be masked by the physiological process of spontaneous recovery. Initially, it was thought that tDCS was capable of altering only the excitability of the cerebral cortex, but recent functional neuroimaging and behavioural studies have also demonstrated tDCS effects on cortical-subcortical functional circuits ${ }^{[1]}$. However, to the best of our knowledge, its efficacy specifically in thalamic aphasia has not previously been investigated.

In this case of subacute thalamic aphasia, we assessed the effectiveness of combined tDCS and SLT. Since the patient showed no improvement in the first 40-day period after the stroke, which is considered the critical period for spontaneous recovery, we hypothesize that tDCS may have stimulated significant cerebral reorganization. As is well known, neuroplasticity is greater during the earlier stages of recovery. The period of maximum neuroplasticity in humans is not entirely clear ${ }^{[5]}$, but it has been suggested that modulation of brain excitability with tDCS is more efficient during the early stages because of neuroprotective and anti-inflammatory mechanisms, and neural growth promotion ${ }^{[6]}$. Our patient showed improvement in linguistic and behavioural communication. This may be due to the fact that the thalamus has a central role in the development of the higher functions, such as memory, behavioural regulation and emotions.

Our results, even though the treatment was applied in the subacute phase, are important for language rehabilitation. The long-term effects of this treatment should be assessed and monitored and our findings investigated further.

\section{REFERENCES}

1. Polanía R, Paulus W, Nitsche MA. Modulating cortico-striatal and thalamo-cortical functional connectivity with transcranial direct current stimulation. Hum Brain Mapp 2012;33(10):2499-2508.

2. Ford AA, Triplett W, Sudhyadhom A, Gullett J, McGregor K, Fitzgerald DB, et al. Broca's area and its striatal and thalamic connections: a diffusion-MRI tractography study. Front Neuroanat 2013;7:8.

3. Llano DA. Functional imaging of the thalamus in language. Brain Lang 2013;126(1):62-72.

4. Biou E, Cassoudesalle H, Cogné M, Sibon I, De Gabory I, Dehail P, et al. Transcranial direct current stimulation in post-stroke aphasia rehabilitation: a systematic review. Ann Phys Rehabil Med 2019;62(2):104-121.

5. Coleman ER, Moudgal R, Lang K, Hyacinth HI, Awosika OO, Kissela BM, et al. Early rehabilitation after stroke: a narrative review. Curr Atheroscler Rep 2017;19(12):59.

6. Pavlova EL, Semenov RV, Guekht AB. Effect of tDCS on fine motor control of patients in subacute and chronic post-stroke stages. J Mot Behav 2020;52(4):383-395. 